\title{
Endoscopic Removal of a Bullet in Rosenmuller Fossa: Case Report
}

\author{
Joshua D. Burks ${ }^{1}$ Chad A. Glenn ${ }^{1}$ Andrew K. Conner ${ }^{1}$ Phillip A. Bonney ${ }^{1}$ Jose A. Sanclement ${ }^{2}$ \\ Michael E. Sughrue ${ }^{1}$
}

1 Department of Neurosurgery, University of Oklahoma Health Sciences Center, Oklahoma City, Oklahoma, United States

2 Department of Otorhinolaryngology, University of Oklahoma Health

Sciences Center, Oklahoma City, Oklahoma, United States

\begin{abstract}
Address for correspondence Michael E. Sughrue, MD, Department of Neurosurgery, University of Oklahoma Health Sciences Center, 1000 North Lincoln Boulevard, Suite 4000, Oklahoma City, OK 73104, United States (e-mail: michael-sughrue@ouhsc.edu).
\end{abstract}

J Neurol Surg Rep 2016;77:e83-e85.
Abstract
Keywords
- Rosenmuller fossa
- skull base
- anterior
- case report
- endoscopic
- nasal septal flap
- endonasal
- pharyngeal recess

Fractures of the anterior skull base may occur in gunshot victims and can result in traumatic cerebrospinal fluid (CSF) leak. Less commonly, CSF leaks occur days or even weeks after the trauma occurred. Here, we present the case of a 21-year-old man with a delayed-onset, traumatic CSF leak secondary to a missile injury that left a bullet fragment in the Rosenmuller fossa. The patient was treated successfully with endoscopic, endonasal extraction of the bullet, and repair with a nasal septal flap. Foreign bodies lodged in Rosenmuller fossa can be successfully treated with endoscopic skull base surgery.

\section{Introduction}

Penetrating trauma of the skull base can occur by any number of mechanisms, but often involves the use of firearms. Fractures at the anterior base of the skull are common in these injuries, ${ }^{1}$ and often result in cerebrospinal fluid (CSF) leaks. The gold standard for diagnosing CSF leak is the $\beta-2$ transferrin test. However, the "halo sign" has also traditionally been used. CSF leaks may be either spontaneous or traumatic, and most traumatic CSF leaks will resolve within 1 week. $^{2}$ In contrast, CSF leak with delayed occurrence has a low likelihood of resolving without surgery. ${ }^{3}$

The fossa of Rosenmuller is also known as the posterolateral recess or the pharyngeal recess. It is formed by nasal sinus mucosal reflections over the longus colli muscle and is located posterior and superior to the ostium of the Eustachian tube. ${ }^{4,5}$

Here, we present the unusual case of delayed-onset, traumatic CSF leak in a young man with a bullet fragment lodged in the fossa of Rosenmuller who underwent nasal endoscopic repair.

received

January 28, 2016 accepted after revision April 5, 2016

\section{History and Examination}

A 21-year-old man presented with rhinorrhea 6 weeks after sustaining a gunshot wound to the face entering through the maxilla. Initially following the injury, he was diagnosed with a fractured mandible, fractured right occipital condyle, and right orbital floor blowout by computed tomography imaging. A retained ballistic fragment located near the left occipital condyle was also identified. A CSF leak was not present during the first hospitalization, and angiographic studies did not reveal arterial injury. Due to the location of the retained fragment and the lack of a CSF leak, the patient was managed conservatively. He was discharged home after further observation. After 6 weeks, the patient presented with rhinorrhea. Imaging at that time revealed the path of the bullet through the right maxilla, the right maxillary sinus traversing the nasopharynx, with the projectile coming to rest just left of the clivus in the pharyngeal recess (fossa of Rosenmuller), as shown in -Fig. 1. Due to the delayed presentation and abundant CSF rhinorrhea, further observation or lumbar
License terms

Stuttgart · New York

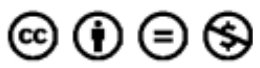




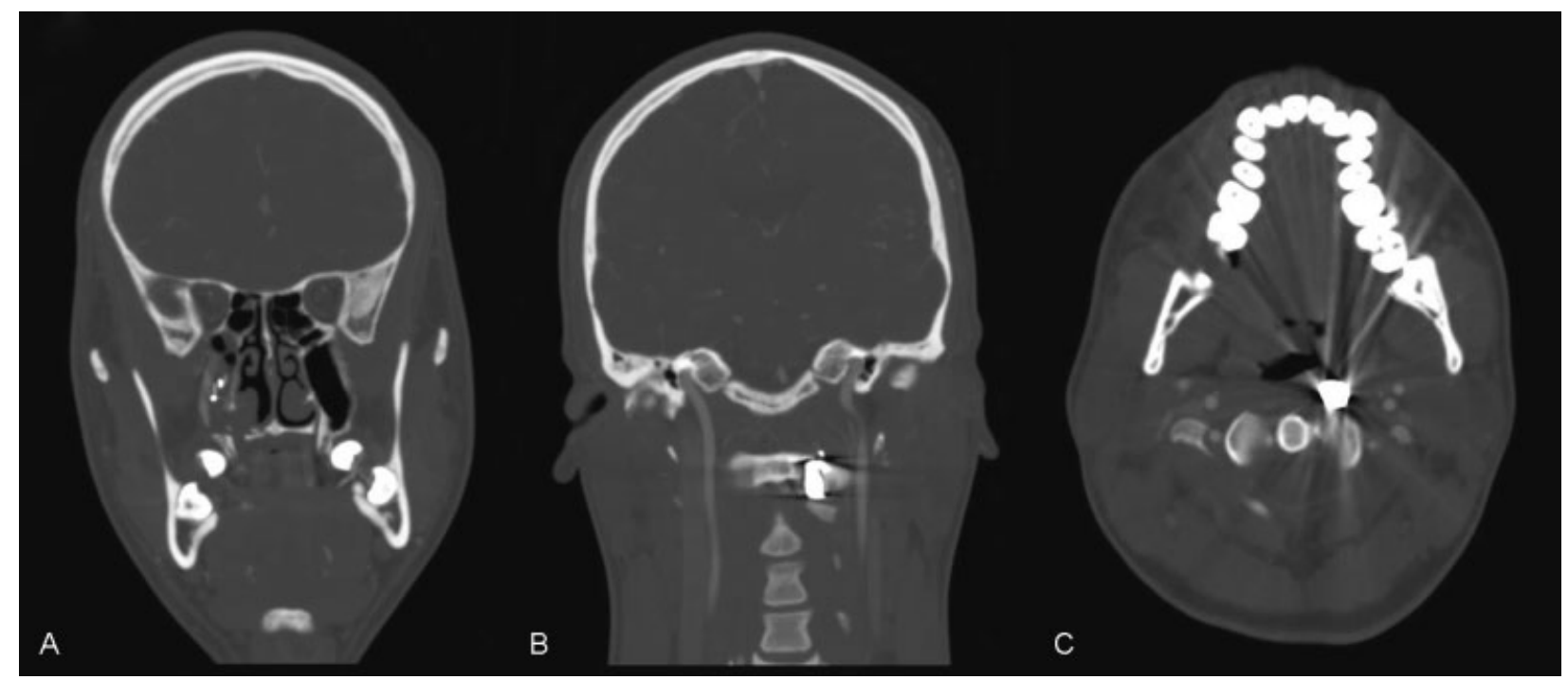

Fig. 1 Computed tomography with angiography demonstrating trajectory of the missile. (A) Coronal image showing the path of the bullet through the maxilary sinus on the right side with residual osseous fragments. (B) Coronal image depicting the bullet resting just left of the clivus in Rosenmuller fossa. Note proximity to the internal carotid artery to Rosenmuller fossa. (C) Axial image at the level of C1, just superior to the bifurcation of the common carotid artery. The internal carotid artery, external carotid artery, and internal jugular vein are appreciated lateral to the bullet.

drain placement were thought to be inadequate. It was felt the CSF leak was likely coming from the skull base near the site of the retained fragment. Nasal endoscopic surgery for exploration and identification of the dural defect was sought.

\section{Operation and Postoperative Course}

A uninostril endonasal approach was performed, including a posterior septectomy, left ethmoidectomy and maxillectomy. A nasoseptal flap was obtained for the repair. The midline heel of the hard palate was drilled to increase the inferior view. After exposing the left pterygoid plate, the scar tissue of pharyngobasilar fascia was divided to expose the Eustachian tube and lower clivus. The Eustachian tube was mobilized laterally and inferiorly, exposing the bullet fragment ( - Fig. 2A). After further dissection and subsequent bullet extraction, a vertical linear defect along the lateral aspect of the clivus down to Rosenmuller fossa was identified ( $\mathbf{F i g . ~ 2 B ) . ~ A ~ m u l t i l a y e r e d ~ r e p a i r ~ c o n s i s t i n g ~}$ of a dural substitute, fibrin glue, and the nasoseptal flap was performed. A lumbar drain was not inserted.

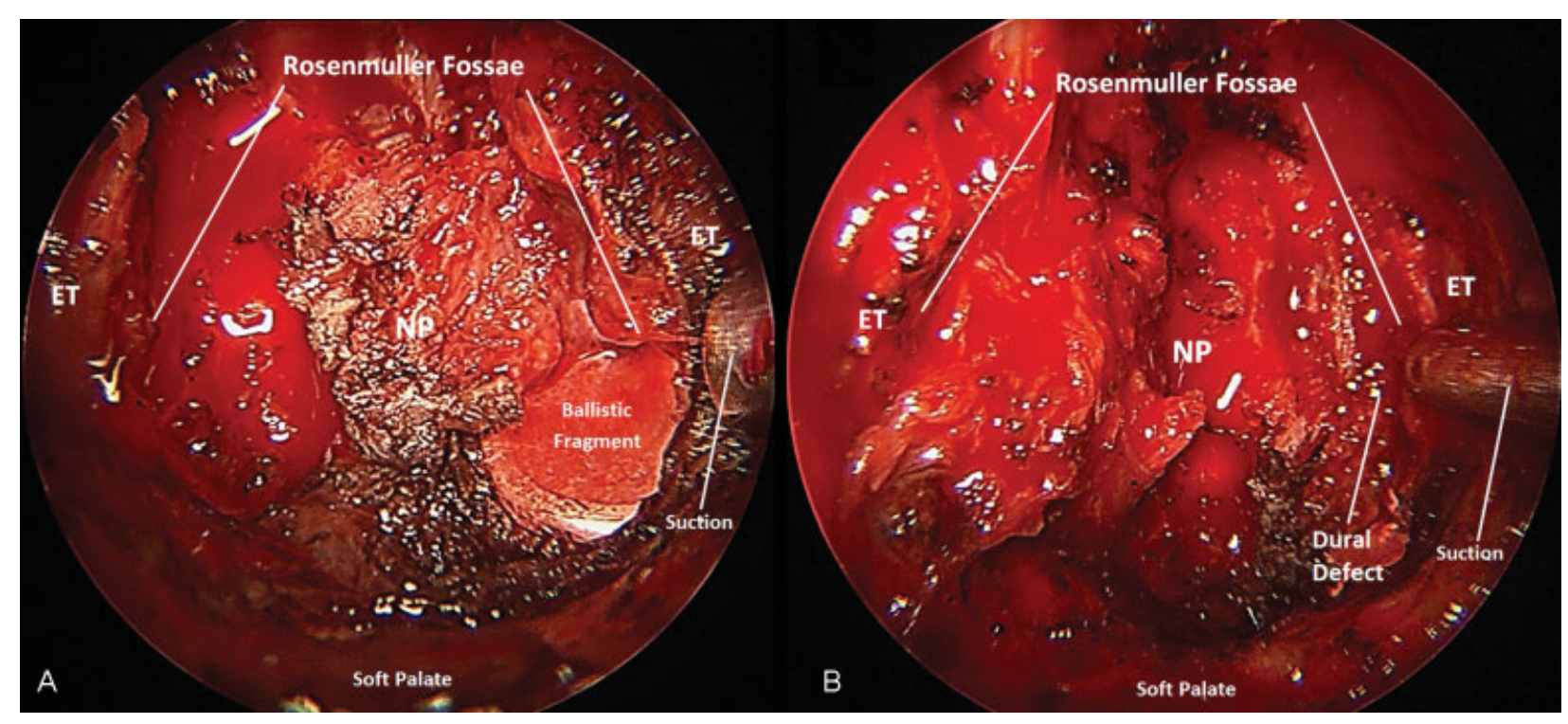

Fig. 2 Endoscopic view. (A) Intraoperative endoscopic view of retained ballistic fragment located within the left fossa of Rosenmuller. The bilateral Eustachian tubes are visualized. Traumatic changes are noted in the nasopharynx and the patient's left Eustachian tube which is displaced laterally by the suction tip. The soft palate is labeled for reference. (B) Intraoperative endoscopic view of dural defect after resection of necrotic tissue and extraction of the ballistic fragment. Cerebrospinal fluid was noted to egress from the dural defect. ET, Eustachian tube; NP, nasopharynx. 
Nasal packing was placed postoperatively and was removed 7 days after surgery. CSF leak was not present immediately postoperatively. There were no postoperative complications, and the patient was discharged on postoperative day 2. As of 3-month follow-up, the patient's CSF leak has not returned.

\section{Discussion}

To our knowledge, our case is the first report of a missile becoming lodged within Rosenmuller fossa, resulting in delayed CSF leak. Operating in this space requires consideration for the position of the internal carotid artery, which is usually situated laterally to the Rosenmuller fossa. There is limited information available on treating this type of injury. A previous case report reviews a delayed traumatic CSF leak from Rosenmuller fossa in a 3-year-old child after a fall. She was found to have a CSF fistula originating from the medial part of the sphenoid, which was repaired successfully with a fascia lata graft at the anterior skull base. ${ }^{6}$ A second, more recent report describes a 57-year-old woman with a bullet lodged within the sphenoid bone who was found to have CSF leak at initial presentation. ${ }^{7}$ The bullet was removed transnasally with endoscopic surgery, and she had no evidence of CSF leak at 6 months follow-up.

While CSF leak following trauma or surgery is far more common than spontaneous CSF leak, it remains relatively rare. Rates of CSF leak following any head trauma have been reported at 2 to $3 \%{ }^{2}$ In a study of 1,278 patients with maxillofacial trauma, 17 (1.3\%) had CSF leak. ${ }^{8}$ All of these patients had skull-base fractures. However, rates of CSF leak are likely higher for patients suffering from missile wounds. In one study from the Vietnam War, of 1,133 men with missile wounds of the brain, 101 (8.9\%) were complicated by CSF fistula. ${ }^{9}$ Current data are lacking regarding the incidence of delayed traumatic CSF leak.

Skull-base CSF leaks are usually repaired with endonasal surgery, and nasoseptal flap(s) are often implemented. Patients treated in this fashion have been shown to have less morbidity. ${ }^{10}$ Other authors have commented on the reliability of the nasoseptal flap to cover a range of skull-base defects as well as its ease of harvesting, making it first-line treatment in such scenarios. ${ }^{11}$ Dual and triple flap techniques that combine various anterior, lateral, and nasoseptal flaps can also be used in extensive and severe cases. $^{12}$

Recent studies aimed at understanding CSF leaks originating at the skull base have focused on risks for recurrence postoperatively. Size of dural defect and location of surgery have been found to be important risk factors for CSF leak in skull-base reconstruction using nasoseptal flaps, with dural defects larger than $2.0 \mathrm{~cm}^{2}$ and surgery to the sella or clivus posing a greater risk. ${ }^{13}$ Despite a heightened risk for postoperative recurrence, the patient's CSF leak had not returned 3 months postoperatively following the multilayered repair.
In conclusion, we present a case of foreign body penetration into Rosenmuller fossa treated successfully with nasal endoscopic surgical repair.

\section{Disclosure}

The authors report no conflict of interest concerning the materials or methods used in this study or the findings specified in this report.

Funding Source

None.

\section{Acknowledgment}

None.

\section{References}

1 Betz P, Stiefel D, Hausmann R, Eisenmenger W. Fractures at the base of the skull in gunshots to the head. Forensic Sci Int 1997; 86(3):155-161

2 Spetzler RF, Zabramski JM. Cerebrospinal fluid fistula. Contemp Neurosurg 1986;8:1-7

3 Greenberg M. Handbook of Neurosurgery. 6th ed. New York, NY: Thieme Medical Publishers; 2006:174-178

4 Chong VF, Fan YF. Radiology of the nasopharynx: pictorial essay. Australas Radiol 2000;44(1):5-13

5 Silver AJ, Mawad ME, Hilal SK, Sane P, Ganti SR. Computed tomography of the nasopharynx and related spaces. Part I: Anatomy. Radiology 1983;147(3):725-731

6 Jaffe B, Welch K, Strand R, Treves S. Cerebrospinal fluid rhinorrhea via the fossa of Rosenmuller. Laryngoscope 1976;86(7):903-907

7 Hatch NU, Riley KO, Woodworth BA. Endoscopic removal of a bullet penetrating the middle cranial fossa. Skull Base Rep 2011; $1(1): 47-50$

8 Hasheminia D, Kalantar Motamedi MR, Hashemzehi H, Nazeri R, Movahedian B. A 7-year study of 1,278 patients with maxillofacial trauma and cerebrospinal fluid leak. J Maxillofac Oral Surg 2015; 14(2):258-262

9 Meirowsky AM, Caveness WF, Dillon JD, et al. Cerebrospinal fluid fistulas complicating missile wounds of the brain. J Neurosurg 1981;54(1):44-48

10 Schoentgen C, Henaux PL, Godey B, Jegoux F. Management of posttraumatic cerebrospinal fluid (CSF) leak of anterior skull base: 10 years experience. Acta Otolaryngol 2013;133(9):944-950

11 El-Sayed IH, Roediger FC, Goldberg AN, Parsa AT, McDermott MW. Endoscopic reconstruction of skull base defects with the nasal septal flap. Skull Base 2008;18(6):385-394

12 Archer JB, Sun H, Bonney PA, et al. Extensive traumatic anterior skull base fractures with cerebrospinal fluid leak: classification and repair techniques using combined vascularized tissue flaps. J Neurosurg 2016;124(3):647-656

13 Gruss CL, Al Komser M, Aghi MK, et al. Risk factors for cerebrospinal leak after endoscopic skull base reconstruction with nasoseptal flap. Otolaryngol Head Neck Surg 2014;151(3):516-521 